\title{
On backward uniqueness for parabolic equations when Osgood continuity of the coefficients fails at one point
}

\section{Daniele Del Santo $^{1}$ (D) $\cdot$ Martino Prizzi $^{1}$}

Received: 22 September 2020 / Accepted: 3 April 2021 / Published online: 17 April 2021

(c) The Author(s) 2021

\section{Abstract}

We prove the uniqueness for backward parabolic equations whose coefficients are Osgood continuous in time for $t>0$ but not at $t=0$.

Keywords Backward parabolic operators · Non-Lipschitz-continuous coefficients ·

Paramultiplication

Mathematics Subject Classification Primary: 35K10 - Secondary: 35S50

\section{Introduction}

Let us consider the following backward parabolic operator

$$
L=\partial_{t}+\sum_{j, k=1}^{n} \partial_{x_{j}}\left(a_{j, k}(t, x) \partial_{x_{k}}\right)+\sum_{j=1}^{n} b_{j}(t, x) \partial_{x_{j}}+c(t, x),
$$

where all the coefficients are assumed to be defined in $[0, T] \times \mathbb{R}^{n}$, measurable and bounded; $\left(a_{j, k}(t, x)\right)_{j, k}$ is a real symmetric matrix for all $(t, x) \in[0, T] \times \mathbb{R}^{n}$ and there exists $\lambda_{0} \in(0,1]$ such that

$$
\sum_{j, k=1}^{n} a_{j, k}(t, x) \xi_{j} \xi_{k} \geq \lambda_{0}|\xi|^{2}
$$

for all $(t, x) \in[0, T] \times \mathbb{R}^{n}$ and $\xi \in \mathbb{R}^{n}$.

Given a functional space $\mathcal{H}$, we say that the operator $L$ has the $\mathcal{H}$-uniqueness property if, whenever $u \in \mathcal{H}, L u=0$ in $[0, T] \times \mathbb{R}^{n}$ and $u(0, x)=0$ in $\mathbb{R}^{n}$, then $u=0$ in $[0, T] \times \mathbb{R}^{n}$.

In the present paper, we are interested in the $\mathcal{H}$-uniqueness property for the operator $L$ defined in (1), when

$$
\mathcal{H}=H^{1}\left((0, T), L^{2}\left(\mathbb{R}^{n}\right)\right) \cap L^{2}\left((0, T), H^{2}\left(\mathbb{R}^{n}\right)\right)
$$

Daniele Del Santo

delsanto@units.it

1 Università di Trieste, Trieste, Italy 
(let us remark that this choice for $\mathcal{H}$ is, in some sense, natural, since, from elliptic regularity results, the domain of the operator $-\sum_{j, k=1}^{n} \partial_{x_{j}}\left(a_{j, k}(t, x) \partial_{x_{k}}\right)$ in $L^{2}\left(\mathbb{R}^{n}\right)$ is $H^{2}\left(\mathbb{R}^{n}\right)$, for all $t \in[0, T])$.

It is well known that, in dealing with the uniqueness property for partial differential operators, one of the main issues is the regularity of the coefficients. For example, in the case of elliptic operators, the uniqueness property in the case of Lipschitz continuous coefficients was proved by Hörmander in [14] (see [17] for a more refined result), while a famous non-uniqueness counterexample, for an elliptic operator having Hölder continuous coefficients, is due to Pliś (see [16]).

In $[9,10]$, we investigated the problem of finding the minimal regularity assumptions on the coefficients $a_{j, k}$ ensuring the $\mathcal{H}$-uniqueness property to (1). Namely, we proved the $\mathcal{H}$ -uniqueness property for (1) when the coefficients $a_{j, k}$ are Lipschitz continuous in $x$ and the regularity in $t$ is given in terms of a modulus of continuity $\mu$, i.e.,

$$
\sup _{\substack{s_{1}, s_{2} \in[0, T], x \in \mathbb{R}^{n}}} \frac{\left|a_{j, k}\left(s_{1}, x\right)-a_{j, k}\left(s_{2}, x\right)\right|}{\mu\left(\left|s_{1}-s_{2}\right|\right)} \leq C,
$$

where $\mu$ satisfies the so-called Osgood condition

$$
\int_{0}^{1} \frac{1}{\mu(s)} \mathrm{d} s=+\infty
$$

A counterexample in [9], similar to that one of Pliś quoted here above, shows that, considering the regularity with respect to $t$ for the $a_{j, k}$, the Osgood condition is sharp: given any non-Osgood modulus of continuity $\mu$, it is possible to construct a backward parabolic operator like (1), whose coefficients are $C^{\infty}$ in $x$ and $\mu$-continuous in $t$, for which the $\mathcal{H}$ -uniqueness property does not hold.

It is interesting to remark that, in the recalled counterexample, the coefficients are in fact $C^{\infty}$ in $t$ for $t \neq 0$, and the Osgood continuity fails only at $t=0$.

The loss of regularity for the coefficients at a single point is widely considered, e.g., in the case of well-posedness in the Cauchy problem for second-order hyperbolic operators of the type

$$
P=\partial_{t}^{2}-\sum_{j, k=1}^{n} \partial_{x_{j}}\left(a_{j, k}(t, x) \partial_{x_{k}}\right)+\sum_{j=1}^{n} b_{j}(t, x) \partial_{x_{j}}+c(t, x),
$$

under the condition (2). For such class of operators, we have the well-posedness in Sobolev spaces when the coefficients are log-Lipschitz continuous with respect to $t$, there exist counterexamples to this property when the Lipschitz continuity fails only at $t=0$, and, finally, the well-posedness in Sobolev spaces can be recovered adding a control on the Lipschitz constant of the $a_{j, k}$ 's, for $t$ going to 0 (the literature on such kind of problems is huge, see, e.g., $[4-8,13,18])$

In this paper, we show that if the loss of the Osgood continuity is properly controlled as $t$ goes to 0 , then the $\mathcal{H}$-uniqueness property for (1) remains valid. Our hypothesis reads as follows: given a modulus of continuity $\mu$ satisfying the Osgood condition, we assume that the coefficients $a_{j, k}$ are Hölder continuous with respect to $t$ on $[0, T]$, and for all $t \in(0, T]$ 


$$
\sup _{\substack{s_{1}, s_{2} \in[t, T], x \in \mathbb{R}^{n}}} \frac{\left|a_{j, k}\left(s_{1}, x\right)-a_{j, k}\left(s_{2}, x\right)\right|}{\mu\left(\left|s_{1}-s_{2}\right|\right)} \leq C t^{-\beta},
$$

where $0<\beta<1$. The coefficients $a_{j, k}$ are assumed to be globally Lipschitz continuous in $x$. Under such hypothesis, we prove that the $\mathcal{H}$-uniqueness property holds for (1). As in [9, 10], the uniqueness result is consequence of a Carleman estimate with a weight function shaped on the modulus of continuity $\mu$. The weight function is obtained as solution of a specific second-order ordinary differential equation. In the previous results cited above, the corresponding o.d.e. is autonomous. Here, on the contrary, the time-dependent control (4) yields to a non-autonomous o.d.e. Also, the "Osgood singularity" of $a_{j, k}$ at $t=0$ introduces a number of new technical difficulties which are not present in the fully Osgood-regular situation considered before.

The result is sharp in the following sense: we exhibit a counterexample in which the coefficients $a_{j, k}$ are Hölder continuous with respect to $t$ on $[0, T]$, for all $t \in(0, T]$ and for all $\epsilon>0$

$$
\sup _{\substack{s_{1}, s_{2} \in[t, T], x \in \mathbb{R}^{n}}} \frac{\left|a_{j, k}\left(s_{1}, x\right)-a_{j, k}\left(s_{2}, x\right)\right|}{\left|s_{1}-s_{2}\right|} \leq C t^{-(1+\epsilon)},
$$

and the operator (1) does not have the $\mathcal{H}$-uniqueness property. The borderline case $\epsilon=0$ in (5) is considered in paper [11]. In such a situation, only a very particular uniqueness result holds and the problem remains essentially open.

\section{Main result}

We start with the definition of modulus of continuity.

Definition 1 A function $\mu:[0,1] \rightarrow[0,1]$ is a modulus of continuity if it is continuous, concave, strictly increasing and $\mu(0)=0, \mu(1)=1$.

Remark 1 Let $\mu$ be a modulus of continuity. Then

- for all $s \in[0,1], \mu(s) \geq s$;

- on $(0,1]$, the function $s \mapsto \frac{\mu(s)}{s}$ is decreasing;

- the $\operatorname{limit}_{\lim _{s \rightarrow 0^{+}}} \frac{\mu(s)}{s}$ exists;

- on $[1,+\infty)$, the function $\sigma \mapsto \sigma \mu\left(\frac{1}{\sigma}\right)$ is increasing;

- on $[1,+\infty)$, the function $\sigma \mapsto \frac{1}{\sigma^{2} \mu\left(\frac{1}{\sigma}\right)}$ is decreasing.

Definition 2 Let $\mu$ be a modulus of continuity and let $\varphi: I \rightarrow B$, where $I$ is an interval in $\mathbb{R}$ and $B$ is a Banach space. $\varphi$ is a function in $C^{\mu}(I, B)$ if $\varphi \in L^{\infty}(I, B)$ and

$$
\|\varphi\|_{C^{\mu}(I, B)}=\|\varphi\|_{L^{\infty}(I, B)}+\sup _{\substack{t, s \in I \\ 0<|t-s|<1}} \frac{\|\varphi(t)-\varphi(s)\|_{B}}{\mu(|t-s|)}<+\infty .
$$


Remark 2 Let $\alpha \in(0,1)$ and $\mu(s)=s^{\alpha}$. Then, $C^{\mu}(I, B)$ is $C^{0, \alpha}(I, B)$, the space of Höldercontinuous functions. Let $\mu(s)=s$. Then, $C^{\mu}(I, B)$ is $\operatorname{Lip}(\mathrm{I}, \mathrm{B})$, the space of bounded Lipschitz-continuous functions.

We introduce the notion of Osgood modulus of continuity.

Definition 3 Let $\mu$ be a modulus of continuity. $\mu$ satisfies the Osgood condition if

$$
\int_{0}^{1} \frac{1}{\mu(s)} \mathrm{d} s=+\infty
$$

Remark 3 Examples of moduli of continuity satisfying the Osgood condition are $\mu(s)=s$ and $\mu(s)=s \log \left(e+\frac{1}{s}-1\right)$.

We state our main result.

Theorem 1 Let $L$ be the operator

$$
L=\partial_{t}+\sum_{j, k=1}^{n} \partial_{x_{j}}\left(a_{j, k}(t, x) \partial_{x_{k}}\right)+\sum_{j=1}^{n} b_{j}(t, x) \partial_{x_{j}}+c(t, x),
$$

where all the coefficients are supposed to be complex valued, defined in $[0, T] \times \mathbb{R}^{n}$, measurable and bounded. Let $\left(a_{j, k}(t, x)\right)_{j, k}$ be a real symmetric matrix and suppose there exists $\lambda_{0} \in(0,1]$ such that

$$
\sum_{j, k=1}^{n} a_{j, k}(t, x) \xi_{j} \xi_{k} \geq \lambda_{0}|\xi|^{2},
$$

for all $(t, x) \in[0, T] \times \mathbb{R}^{n}$ and for all $\xi \in \mathbb{R}^{n}$. Under this condition, L is a backward parabolic operator. Let $\mathcal{H}$ be the space of functions such that

$$
\mathcal{H}=H^{1}\left((0, T), L^{2}\left(\mathbb{R}^{n}\right)\right) \cap L^{2}\left((0, T), H^{2}\left(\mathbb{R}^{n}\right)\right) .
$$

Let $\mu$ be a modulus of continuity satisfying the Osgood condition. Suppose that there exist $\alpha \in(0,1)$ and $C>0$ such that,

i) for all $j, k=1, \ldots, n$,

$$
a_{j, k} \in C^{0, \alpha}\left([0, T], L^{\infty}\left(\mathbb{R}^{n}\right)\right) \cap L^{\infty}\left([0, T], \operatorname{Lip}\left(\mathbb{R}^{n}\right)\right) ;
$$

ii) for all $j, k=1, \ldots, n$ and for all $t \in(0, T]$,

$$
\sup _{\substack{s_{1}, s_{2} \in[t, T], x \in \mathbb{R}^{n}}} \frac{\left|a_{j, k}\left(s_{1}, x\right)-a_{j, k}\left(s_{2}, x\right)\right|}{\mu\left(\left|s_{1}-s_{2}\right|\right)} \leq C t^{\alpha-1} .
$$

Then $L$ has the $\mathcal{H}$-uniqueness property, i.e., if $u \in \mathcal{H}, L u=0$ in $[0, T] \times \mathbb{R}^{n}$ and $u(0, x)=0$ in $\mathbb{R}^{n}$, then $u=0$ in $[0, T] \times \mathbb{R}^{n}$. 
Remark 4 The hypothesis (10), in particular the Hölder regularity with respect to $t$, is due to technical requirement for obtaining the Carleman estimate from which the main result is deduced. It does not seem easy to substitute it with different or weaker conditions.

\section{Weight function and Carleman estimate}

Defining

$$
\phi(t)=\int_{\frac{1}{t}}^{1} \frac{1}{\mu(s)} \mathrm{d} s,
$$

the function $\phi$ is a strictly increasing $C^{1}$ function on $[1,+\infty)$, with values in $[0,+\infty)$, and, by the Osgood condition, it is bijective. Moreover, for all $t \in[1,+\infty)$,

$$
\phi^{\prime}(t)=\frac{1}{t^{2} \mu\left(\frac{1}{t}\right)} .
$$

We remark that $\phi^{\prime}(1)=1$ and $\phi^{\prime}$ is decreasing in $[1,+\infty)$, so that $\phi$ is a concave function. Moreover, we notice also that $\phi^{-1}:[0,+\infty) \rightarrow[1,+\infty)$ and, for all $s \in[0,+\infty)$,

$$
\phi^{-1}(s) \geq 1+s \text {. }
$$

We define

$$
\psi_{\gamma}(\tau)=\phi^{-1}\left(\gamma \int_{0}^{\frac{\tau}{\gamma}}(T-s)^{\alpha-1} \mathrm{~d} s\right)
$$

where $\tau \in[0, \gamma T]$.

$$
\phi\left(\psi_{\gamma}(\tau)\right)=\gamma \int_{0}^{\frac{\tau}{\gamma}}(T-s)^{\alpha-1} \mathrm{~d} s
$$

and

$$
\phi^{\prime}\left(\psi_{\gamma}(\tau)\right) \psi_{\gamma}^{\prime}(\tau)=\left(T-\frac{\tau}{\gamma}\right)^{\alpha-1}
$$

Then

$$
\psi_{\gamma}^{\prime}(\tau)=\left(T-\frac{\tau}{\gamma}\right)^{\alpha-1} \cdot\left(\psi_{\gamma}(\tau)\right)^{2} \mu\left(\frac{1}{\psi_{\gamma}(\tau)}\right),
$$

i. e. $\psi_{\gamma}$ is a solution to the differential equation

$$
u^{\prime}(\tau)=\left(T-\frac{\tau}{\gamma}\right)^{\alpha-1} u^{2}(\tau) \mu\left(\frac{1}{u(\tau)}\right) .
$$

Finally we set, for $\tau \in[0, \gamma T]$, 


$$
\Phi_{\gamma}(\tau)=\int_{0}^{\tau} \psi_{\gamma}(\sigma) \mathrm{d} \sigma
$$

Remark that, with this definition, $\Phi^{\prime}(\tau)=\psi_{\gamma}(\tau)$ and

$$
\Phi_{\gamma}^{\prime \prime}(\tau)=\left(T-\frac{\tau}{\gamma}\right)^{\alpha-1}\left(\Phi_{\gamma}^{\prime}(\tau)\right)^{2} \mu\left(\frac{1}{\Phi_{\gamma}^{\prime}(\tau)}\right) .
$$

In particular, for $t \in\left[0, \frac{T}{2}\right]$,

$$
\Phi_{\gamma}^{\prime \prime}(\gamma(T-t))=t^{\alpha-1} \Phi_{\gamma}^{\prime}(\gamma(T-t)) \frac{\mu\left(\frac{1}{\Phi_{\gamma}^{\prime}(\gamma(T-t))}\right)}{\frac{1}{\Phi_{\gamma}^{\prime}(\gamma(T-t))}} \geq t^{\alpha-1} \geq\left(\frac{T}{2}\right)^{\alpha-1},
$$

since $\Phi_{\gamma}^{\prime}(\gamma(T-t))=\psi_{\gamma}(\gamma(T-t)) \geq 1$ and $\frac{\mu(s)}{s} \geq 1$ for all $s \in(0,1]$.

We can now state the Carleman estimate.

Theorem 2 In the previous hypotheses, there exist $\gamma_{0}>0, C>0$ such that

$$
\begin{aligned}
& \int_{0}^{\frac{T}{2}} e^{\frac{2}{\gamma} \Phi_{\gamma}(\gamma(T-t))}\left\|\partial_{t} u+\sum_{j, k=1}^{n} \partial_{x_{j}}\left(a_{j, k}(t, x) \partial_{x_{k}} u\right)\right\|_{L^{2}}^{2} \mathrm{~d} t \\
& \geq C \gamma^{\frac{1}{2}} \int_{0}^{\frac{T}{2}} e^{\frac{2}{\gamma} \Phi_{\gamma}(\gamma(T-t))}\left(\left\|\nabla_{x} u\right\|_{L^{2}}^{2}+\gamma^{\frac{1}{2}}\|u\|_{L^{2}}^{2}\right) \mathrm{d} t
\end{aligned}
$$

for all $\gamma>\gamma_{0}$ and for all $u \in C_{0}^{\infty}\left(\mathbb{R}^{n+1}\right)$ such that $\operatorname{Supp} u \subseteq\left[0, \frac{T}{2}\right] \times \mathbb{R}^{n}$.

The way of obtaining the $\mathcal{H}$-uniqueness from the inequality (17) is a standard procedure, the details of which can be found in [9, Par. 3.4].

\section{Proof of the Carleman estimate}

\subsection{Littlewood-Paley decomposition}

We will use the so-called Littlewood-Paley theory. We refer to [2, 3, 15] and [1] for the details. Let $\psi \in C^{\infty}([0,+\infty), \mathbb{R})$ such that $\psi$ is non-increasing and

$$
\psi(t)=1 \quad \text { for } \quad 0 \leq t \leq \frac{11}{10}, \quad \psi(t)=0 \quad \text { for } t \geq \frac{19}{10} .
$$

We set, for $\xi \in \mathbb{R}^{n}$,

$$
\chi(\xi)=\psi(|\xi|), \quad \varphi(\xi)=\chi(\xi)-\chi(2 \xi) .
$$

Given a tempered distribution $u$, the dyadic blocks are defined by

$$
\begin{aligned}
& u_{0}=\Delta_{0} u=\chi(D) u=\mathcal{F}^{-1}(\chi(\xi) \hat{u}(\xi)), \\
& u_{j}=\Delta_{j} u=\varphi\left(2^{-j} D\right) u=\mathcal{F}^{-1}\left(\varphi\left(2^{-j} \xi\right) \hat{u}(\xi)\right) \quad \text { if } \quad j \geq 1,
\end{aligned}
$$


where we have denoted by $\mathcal{F}^{-1}$ the inverse of the Fourier transform. We introduce also the operator

$$
S_{k} u=\sum_{j=0}^{k} \Delta_{j} u=\mathcal{F}^{-1}\left(\chi\left(2^{-k} \xi\right) \hat{u}(\xi)\right) .
$$

We recall some well-known facts on Littlewood-Paley deposition.

Proposition 1 ([8, Prop. 3.1]) Let $s \in \mathbb{R}$. A temperate distribution $u$ is in $H^{s}$ if and only if, for all $j \in \mathbb{N}, \Delta_{j} u \in L^{2}$ and

$$
\sum_{j=0}^{+\infty} 2^{2 j s}\left\|\Delta_{j} u\right\|_{L^{2}}^{2}<+\infty
$$

Moreover, there exists $C>1$, depending only on $n$ and $s$, such that, for all $u \in H^{s}$,

$$
\frac{1}{C} \sum_{j=0}^{+\infty} 2^{2 j s}\left\|\Delta_{j} u\right\|_{L^{2}}^{2} \leq\|u\|_{H^{s}}^{2} \leq C \sum_{j=0}^{+\infty} 2^{2 j s}\left\|\Delta_{j} u\right\|_{L^{2}}^{2} .
$$

Proposition 2 ([12, Lemma 3.2]). A bounded function a is a Lipschitz-continuous function if and only if

$$
\sup _{k \in \mathbb{N}}\left\|\nabla\left(S_{k} a\right)\right\|_{L^{\infty}}<+\infty .
$$

Moreover, there exists $C>0$, depending only on $n$, such that, for all $a \in$ Lip and for all $k \in \mathbb{N}$,

$$
\left\|\Delta_{k} a\right\|_{L^{\infty}} \leq C 2^{-k}\|a\|_{\text {Lip }} \quad \text { and } \quad\left\|\nabla\left(S_{k} a\right)\right\|_{L^{\infty}} \leq C\|a\|_{\text {Lip }},
$$

where $\|a\|_{\text {Lip }}=\|a\|_{L^{\infty}}+\|\nabla a\|_{L^{\infty}}$.

\subsection{Modified Bony's paraproduct}

Definition 4 Let $m \in \mathbb{N} \backslash\{0\}, a \in L^{\infty}$ and $s \in \mathbb{R}$. For all $u \in H^{s}$, we define

$$
T_{a}^{m} u=S_{m-1} a S_{m+1} u+\sum_{k=m-1}^{+\infty} S_{k} a \Delta_{k+3} u .
$$

We recall some known facts on modified Bony's paraproduct.

Proposition 3 ([15, Prop. 5.2.1 and Th. 5.2.8]). Let $m \in \mathbb{N} \backslash\{0\}, a \in L^{\infty}$ and $s \in \mathbb{R}$.

Then $T_{a}^{m}$ maps $H^{s}$ into $H^{s}$ and there exists $C>0$ depending only on $n, m$ and $s$, such that, for all $u \in H^{s}$,

$$
\left\|T_{a}^{m} u\right\|_{H^{s}} \leq C\|a\|_{L^{\infty}}\|u\|_{H^{s}} .
$$

Let $m \in \mathbb{N} \backslash\{0\}$ and let $a \in$ Lip. 
Then $a-T_{a}^{m}$ maps $L^{2}$ into $H^{1}$ and there exists $C^{\prime}>0$ depending only on $n, m$, such that, for all $u \in L^{2}$,

$$
\left\|a u-T_{a}^{m} u\right\|_{H^{1}} \leq C^{\prime}\|a\|_{L i p}\|u\|_{L^{2}} .
$$

Proposition 4 ([8, Cor. 3.12]) Let $m \in \mathbb{N} \backslash\{0\}$ and $a \in$ Lip. Suppose that, for all $x \in \mathbb{R}^{n}$, $a(x) \geq \lambda_{0}>0$.

Then, there exists $m$ depending on $\lambda_{0}$ and $\|a\|_{\text {Lip }}$ such that for all $u \in L^{2}$,

$$
\operatorname{Re}\left\langle T_{a}^{m} u, u\right\rangle_{L^{2}, L^{2}} \geq \frac{\lambda_{0}}{2}\|u\|_{L^{2}} .
$$

A similar result remains valid when $u$ is a vector valued function and $a$ is replaced by $a$ positive definite matrix $\left(a_{j, k}\right)_{j, k}$.

Proposition 5 ([8, Prop. 3.8 and Prop. 3.11] and [10, Prop. 3.8]) Let $m \in \mathbb{N} \backslash\{0\}$ and $a \in \operatorname{Lip}$. Let $\left(T_{a}^{m}\right)^{*}$ be the adjoint operator of $T_{a}^{m}$.

Then, there exists $C>0$ depending only on $n$ and $m$ such that for all $u \in L^{2}$,

$$
\left\|\left(T_{a}^{m}-\left(T_{a}^{m}\right)^{*}\right) \partial_{x_{j}} u\right\|_{L^{2}} \leq C\|a\|_{\text {Lip }}\|u\|_{L^{2}} .
$$

We end this subsection with a property which will needed in the proof of the Carleman estimate.

Proposition 6 ([10, Prop. 3.8]) Let $m \in \mathbb{N} \backslash\{0\}$ and let $a \in$ Lip. Denote by $\left[\Delta_{k}, T_{a}^{m}\right]$ the commutator between $\Delta_{k}$ and $T_{a}^{m}$.

Then, there exists $C>0$ depending only on $n$ and $m$ such that for all $u \in H^{1}$,

$$
\left(\sum_{h=0}^{+\infty}\left\|\partial_{x_{j}}\left(\left[\Delta_{k}, T_{a}^{m}\right] \partial_{x_{k}} u\right)\right\|_{L^{2}}^{2}\right)^{\frac{1}{2}} \leq C\|a\|_{\text {Lip }}\|u\|_{H^{1}} .
$$

\subsection{Approximated Carleman estimate}

Setting

$$
v(t, x)=e^{\frac{1}{\gamma} \Phi_{\gamma}(\gamma(T-t))} u(t, x),
$$

the Carleman estimate (17) becomes: there exist $\gamma_{0}>0, C>0$ such that

$$
\begin{aligned}
& \int_{0}^{\frac{T}{2}}\left\|\partial_{t} v+\sum_{j, k=1}^{n} \partial_{x_{j}}\left(a_{j, k}(t, x) \partial_{x_{k}} v\right)+\Phi_{\gamma}^{\prime}(\gamma(T-t)) v\right\|_{L^{2}}^{2} \mathrm{~d} t \\
& \geq C \gamma^{\frac{1}{2}} \int_{0}^{\frac{T}{2}}\left(\left\|\nabla_{x} v\right\|_{L^{2}}^{2}+\gamma^{\frac{1}{2}}\|u\|_{L^{2}}^{2}\right) \mathrm{d} t,
\end{aligned}
$$

for all $\gamma>\gamma_{0}$ and for all $v \in C_{0}^{\infty}\left(\mathbb{R}^{n+1}\right)$ such that Supp $u \subseteq\left[0, \frac{T}{2}\right] \times \mathbb{R}_{x}^{n}$. 
First of all, using Proposition 4, we fix a value for $m$ in such a way that

$$
\operatorname{Re} \sum_{j, k}\left\langle T_{a_{j, k}}^{m} \partial_{x_{k}} v, \partial_{x_{j}} v\right\rangle_{L^{2}, L^{2}} \geq \frac{\lambda_{0}}{2}\left\|\nabla_{x} v\right\|_{L^{2}}
$$

for all $v \in C_{0}^{\infty}\left(\mathbb{R}^{n+1}\right)$ such that $\operatorname{Supp} u \subseteq\left[0, \frac{T}{2}\right] \times \mathbb{R}^{n}$. Next we use Proposition 3 and in particular from (22) we deduce that (26) will be a consequence of

$$
\begin{aligned}
& \int_{0}^{\frac{T}{2}}\left\|\partial_{t} v+\sum_{j, k=1}^{n} \partial_{x_{j}}\left(T_{a_{j, k}}^{m} \partial_{x_{k}} v\right)+\Phi_{\gamma}^{\prime}(\gamma(T-t)) v\right\|_{L^{2}}^{2} d t \\
& \geq C \gamma^{\frac{1}{2}} \int_{0}^{\frac{T}{2}}\left(\left\|\nabla_{x} v\right\|_{L^{2}}^{2}+\gamma^{\frac{1}{2}}\|u\|_{L^{2}}^{2}\right) d t,
\end{aligned}
$$

since the difference between (26) and (28) is absorbed by the right side part of (28) with possibly a different value of $C$ and $\gamma_{0}$. With a similar argument, using (19) and (25), (28) will be deduced from

$$
\begin{aligned}
& \int_{0}^{\frac{T}{2}} \sum_{h=0}^{+\infty}\left\|\partial_{t} v_{h}+\sum_{j, k=1}^{n} \partial_{x_{j}}\left(T_{a_{j, k}}^{m} \partial_{x_{k}} v_{h}\right)+\Phi_{\gamma}^{\prime}(\gamma(T-t)) v_{h}\right\|_{L^{2}}^{2} \mathrm{~d} t \\
& \geq C \gamma^{\frac{1}{2}} \int_{0}^{\frac{T}{2}} \sum_{h=0}^{+\infty}\left(\left\|\nabla_{x} v_{h}\right\|_{L^{2}}^{2}+\gamma^{\frac{1}{2}}\left\|v_{h}\right\|_{L^{2}}^{2}\right) \mathrm{d} t
\end{aligned}
$$

where we have denoted by $v_{h}$ the dyadic block $\Delta_{h} v$.

We fix our attention on each of the terms

$$
\int_{0}^{\frac{T}{2}}\left\|\partial_{t} v_{h}+\sum_{j, k=1}^{n} \partial_{x_{j}}\left(T_{a_{j, k}}^{m} \partial_{x_{k}} v_{h}\right)+\Phi_{\gamma}^{\prime}(\gamma(T-t)) v_{h}\right\|_{L^{2}}^{2} \mathrm{~d} t
$$

We have

$$
\begin{aligned}
& \int_{0}^{\frac{T}{2}}\left\|\partial_{t} v_{h}+\sum_{j, k=1}^{n} \partial_{x_{j}}\left(T_{a_{j, k}}^{m} \partial_{x_{k}} v_{h}\right)+\Phi_{\gamma}^{\prime}(\gamma(T-t)) v_{h}\right\|_{L^{2}}^{2} \mathrm{~d} t \\
& =\int_{0}^{\frac{T}{2}}\left(\left\|\partial_{t} v_{h}\right\|_{L^{2}}^{2}+\left\|\sum_{j, k=1}^{n} \partial_{x_{j}}\left(T_{a_{j, k}}^{m} \partial_{x_{k}} v_{h}\right)+\Phi_{\gamma}^{\prime}(\gamma(T-t)) v_{h}\right\|_{L^{2}}^{2}\right. \\
& \left.\quad+\gamma \Phi_{\gamma}^{\prime \prime}(\gamma(T-t))\left\|v_{h}\right\|_{L^{2}}^{2}+2 \operatorname{Re}\left\langle\partial_{t} v_{h}, \sum_{j, k=1}^{n} \partial_{x_{j}}\left(T_{a_{j, k}}^{m} \partial_{x_{k}} v_{h}\right)\right\rangle_{L^{2}, L^{2}}\right) \mathrm{d} t
\end{aligned}
$$

Let consider the last term in (30). We define, for $\varepsilon \in\left[0, \frac{T}{2}\right]$,

$$
\tilde{a}_{j, k, \varepsilon}(t, x)= \begin{cases}a_{j, k}(T, x), & \text { if } t \geq T \text { and } x \in \mathbb{R}^{n}, \\ a_{j, k}(t, x), & \text { if } \varepsilon \leq t \leq T \text { and } x \in \mathbb{R}^{n}, \\ a_{j, k}(\varepsilon, x), & \text { if } t<\varepsilon \text { and } x \in \mathbb{R}^{n},\end{cases}
$$

and 


$$
a_{j, k, \varepsilon}(t, x)=\int_{-\varepsilon}^{\varepsilon} \rho_{\varepsilon}(s) \tilde{a}_{j, k, \varepsilon}(t-s, x) d s,
$$

where $\rho \in C_{0}^{\infty}(\mathbb{R})$ with Supp $\rho \subseteq[-1,1], \int_{\mathbb{R}} \rho(s) d s=1, \rho(s) \geq 0$ and $\rho_{\varepsilon}(s)=\frac{1}{\varepsilon} \rho\left(\frac{s}{\varepsilon}\right)$. With a straightforward computation, from (10) and (11), we obtain

$$
\left|a_{j, k}(t, x)-a_{j, k, \varepsilon}(t, x)\right| \leq C \min \left\{\varepsilon^{\alpha}, t^{\alpha-1} \mu(\varepsilon)\right\},
$$

and

$$
\left|\partial_{t} a_{j, k, \varepsilon}(t, x)\right| \leq C \min \left\{\varepsilon^{\alpha-1}, t^{\alpha-1} \frac{\mu(\varepsilon)}{\varepsilon}\right\}
$$

for all $j, k=1 \ldots, n$ and for all $(t, x) \in\left[0, \frac{T}{2}\right] \times \mathbb{R}^{n}$. We deduce

$$
\begin{aligned}
& \int_{0}^{\frac{T}{2}} 2 \operatorname{Re}\left\langle\partial_{t} v_{h}, \sum_{j, k=1}^{n} \partial_{x_{j}}\left(T_{a_{j, k}}^{m} \partial_{x_{k}} v_{h}\right)\right\rangle_{L^{2}, L^{2}} \mathrm{~d} t \\
& =-2 \operatorname{Re} \int_{0}^{\frac{T}{2}} \sum_{j, k=1}^{n}\left\langle\partial_{x_{j}} \partial_{t} v_{h}, T_{a_{j, k}}^{m} \partial_{x_{k}} v_{h}\right\rangle_{L^{2}, L^{2}} \mathrm{~d} t \\
& =-2 \operatorname{Re} \int_{0}^{\frac{T}{2}} \sum_{j, k=1}^{n}\left\langle\partial_{x_{j}} \partial_{t} v_{h},\left(T_{a_{j, k}}^{m}-T_{a_{j, k, \varepsilon}}^{m}\right) \partial_{x_{k}} v_{h}\right\rangle_{L^{2}, L^{2}} \mathrm{~d} t \\
& -2 \operatorname{Re} \int_{0}^{\frac{T}{2}} \sum_{j, k=1}^{n}\left\langle\partial_{x_{j}} \partial_{t} v_{h}, T_{a_{j, k, \varepsilon}}^{m} \partial_{x_{k}} v_{h}\right\rangle_{L^{2}, L^{2}} \mathrm{~d} t .
\end{aligned}
$$

Now, $T_{a_{j, k}}^{m}-T_{a_{j, k, \varepsilon}}^{m}=T_{a_{j, k}-a_{j, k, \varepsilon}}^{m}$ and, from (21) and (31),

$$
\begin{aligned}
\left\|\left(T_{a_{j, k}}^{m}-T_{a_{j, k, \varepsilon}}^{m}\right) \partial_{x_{k}} v_{h}\right\|_{L^{2}} & =\left\|T_{a_{j, k}-a_{j, k, \varepsilon}} \partial_{x_{k}} v_{h}\right\|_{L^{2}} \\
& \leq C \min \left\{\varepsilon^{\alpha}, t^{\alpha-1} \mu(\varepsilon)\right\}\left\|\partial_{x_{k}} v_{h}\right\|_{L^{2}} .
\end{aligned}
$$

Moreover $\left\|\partial_{x_{j}} v_{h}\right\|_{L^{2}} \leq 2^{h+1}\left\|v_{h}\right\|_{L^{2}}$ and $\left\|\partial_{x_{j}} \partial_{t} v_{h}\right\|_{L^{2}} \leq 2^{h+1}\left\|\partial_{t} v_{h}\right\|_{L^{2}}$, so that

$$
\begin{aligned}
& \left|2 \operatorname{Re} \int_{0}^{\frac{T}{2}} \sum_{j, k=1}^{n}\left\langle\partial_{x_{j}} \partial_{t} v_{h},\left(T_{a_{j, k}}^{m}-T_{a_{j, k, \varepsilon}}^{m}\right) \partial_{x_{k}} v_{h}\right\rangle_{L^{2}, L^{2}} d t\right| \\
& \quad \leq 2 C \int_{0}^{\frac{T}{2}} \min \left\{\varepsilon^{\alpha}, t^{\alpha-1} \mu(\varepsilon)\right\} \sum_{j, k=1}^{n}\left\|\partial_{x_{j}} \partial_{t} v_{h}\right\|_{L^{2}}\left\|\partial_{x_{k}} v_{h}\right\|_{L^{2}} d t \\
& \quad \leq \frac{C}{N} \int_{0}^{\frac{T}{2}}\left\|\partial_{t} v_{h}\right\|_{L^{2}}^{2} d t+C N 2^{4(h+1)} \int_{0}^{\frac{T}{2}} \min \left\{\varepsilon^{\alpha}, t^{\alpha-1} \mu(\varepsilon)\right\}\left\|v_{h}\right\|_{L^{2}}^{2} \mathrm{~d} t,
\end{aligned}
$$

where $C$ depends only on $n, m$ and $\left\|a_{j, k}\right\|_{L^{\infty}}$ and $N>0$ can be chosen arbitrarily.

Similarly 


$$
\begin{aligned}
-2 \operatorname{Re} & \int_{0}^{\frac{T}{2}} \sum_{j, k=1}^{n}\left\langle\partial_{x_{j}} \partial_{t} v_{h}, T_{a_{j, k, \varepsilon}}^{m} \partial_{x_{k}} v_{h}\right\rangle_{L^{2}, L^{2}} \mathrm{~d} t \\
= & \int_{0}^{\frac{T}{2}} \sum_{j, k=1}^{n}\left\langle\partial_{x_{j}} v_{h}, T_{\partial_{t} a_{j, k, \varepsilon}}^{m} \partial_{x_{k}} v_{h}\right\rangle_{L^{2}, L^{2}} \mathrm{~d} t \\
& +\int_{0}^{\frac{T}{2}} \sum_{j, k=1}^{n}\left\langle\partial_{x_{j}} v_{h},\left(T_{a_{j, k, \varepsilon}}^{m}-\left(T_{a_{j, k, \varepsilon}}^{m}\right)^{*}\right) \partial_{x_{k}} \partial_{t} v_{h}\right\rangle_{L^{2}, L^{2}} \mathrm{~d} t .
\end{aligned}
$$

From (21) and (32), we have

$$
\begin{aligned}
& \left|\int_{0}^{\frac{T}{2}} \sum_{j, k=1}^{n}\left\langle\partial_{x_{j}} v_{h}, T_{\partial_{t} a_{j, k, \varepsilon}}^{m} \partial_{x_{k}} v_{h}\right\rangle_{L^{2}, L^{2}} d t\right| \\
& \quad \leq C 2^{2(h+1)} \int_{0}^{\frac{T}{2}} \min \left\{\varepsilon^{\alpha-1}, t^{\alpha-1} \frac{\mu(\varepsilon)}{\varepsilon}\right\}\left\|v_{h}\right\|_{L^{2}}^{2} d t,
\end{aligned}
$$

and, from (24),

$$
\begin{aligned}
& \left|\int_{0}^{\frac{T}{2}} \sum_{j, k=1}^{n}\left\langle\partial_{x_{j}} v_{h},\left(T_{a_{j, k, \varepsilon}}^{m}-\left(T_{a_{j, k, \varepsilon}}^{m}\right)^{*}\right) \partial_{x_{k}} \partial_{t} v_{h}\right\rangle_{L^{2}, L^{2}} d t\right| \\
& \quad \leq C \int_{0}^{\frac{T}{2}}\left\|\nabla v_{h}\right\|_{L^{2}}\left\|\partial_{t} v_{h}\right\|_{L^{2}} d t \\
& \quad \leq \frac{C}{N} \int_{0}^{\frac{T}{2}}\left\|\partial_{t} v_{h}\right\|_{L^{2}}^{2} d t+C N 2^{2(h+1)} \int_{0}^{\frac{T}{2}}\left\|v_{h}\right\|_{L^{2}}^{2} d t,
\end{aligned}
$$

where $C$ depends only on $n, m$ and $\left\|a_{j, k}\right\|_{L i p}$ and $N>0$ can be chosen arbitrarily.

As a conclusion, from (30), we finally obtain

$$
\begin{aligned}
\int_{0}^{\frac{T}{2}}\left\|\partial_{t} v_{h}+\sum_{j, k=1}^{n} \partial_{x_{j}}\left(T_{a_{j, k}}^{m} \partial_{x_{k}} v_{h}\right)+\Phi_{\gamma}^{\prime}(\gamma(T-t)) v_{h}\right\|_{L^{2}}^{2} \mathrm{~d} t \\
\geq \int_{0}^{\frac{T}{2}}\left(\left\|\sum_{j, k=1}^{n} \partial_{x_{j}}\left(T_{a_{j, k}}^{m} \partial_{x_{k}} v_{h}\right)+\Phi_{\gamma}^{\prime}(\gamma(T-t)) v_{h}\right\|_{L^{2}}^{2}\right. \\
\quad+\gamma \Phi_{\gamma}^{\prime \prime}(\gamma(T-t))\left\|v_{h}\right\|_{L^{2}}^{2}-C\left(2^{4(h+1)} \min \left\{\varepsilon^{\alpha}, t^{\alpha-1} \mu(\varepsilon)\right\}\right. \\
\left.\left.\quad+2^{2(h+1)}\left(\min \left\{\varepsilon^{\alpha-1}, t^{\alpha-1} \frac{\mu(\varepsilon)}{\varepsilon}\right\}+1\right)\right)\left\|v_{h}\right\|_{L^{2}}^{2}\right) \mathrm{d} t .
\end{aligned}
$$

\subsection{End of the proof}

We start considering (33) for $h=0$. We fix $\varepsilon=\frac{1}{2}$. Recalling (16) we have 


$$
\begin{aligned}
\int_{0}^{\frac{T}{2}} \| \partial_{t} v_{0} & +\sum_{j, k=1}^{n} \partial_{x_{j}}\left(T_{a_{j, k}}^{m} \partial_{x_{k}} v_{0}\right)+\Phi_{\gamma}^{\prime}(\gamma(T-t)) v_{0} \|_{L^{2}}^{2} d t \\
& \geq \int_{0}^{\frac{T}{2}}\left(\gamma \Phi_{\gamma}^{\prime \prime}(\gamma(T-t))-C^{\prime}\right)\left\|v_{0}\right\|_{L^{2}}^{2} \\
& \geq \int_{0}^{\frac{T}{2}}\left(\gamma\left(\frac{T}{2}\right)^{\alpha-1}-C^{\prime}\right)\left\|v_{0}\right\|_{L^{2}}^{2} d t
\end{aligned}
$$

Choosing a suitable $\gamma_{0}$, we have that, for all $\gamma>\gamma_{0}$,

$$
\int_{0}^{\frac{T}{2}}\left\|\partial_{t} v_{0}+\sum_{j, k=1}^{n} \partial_{x_{j}}\left(T_{a_{j, k}}^{m} \partial_{x_{k}} v_{0}\right)+\Phi_{\gamma}^{\prime}(\gamma(T-t)) v_{0}\right\|_{L^{2}}^{2} d t \geq \frac{\gamma}{2} \int_{0}^{\frac{T}{2}}\left\|v_{0}\right\|_{L^{2}}^{2} d t .
$$

We consider (33) for $h \geq 1$. Choosing $\varepsilon=2^{-2 h}$, we have

$$
\begin{aligned}
\int_{0}^{\frac{T}{2}}\left\|\partial_{t} v_{h}+\sum_{j, k=1}^{n} \partial_{x_{j}}\left(T_{a_{j, k}}^{m} \partial_{x_{k}} v_{h}\right)+\Phi_{\gamma}^{\prime}(\gamma(T-t)) v_{h}\right\|_{L^{2}}^{2} d t \\
\geq \int_{0}^{\frac{T}{2}}\left(\left\|\sum_{j, k=1}^{n} \partial_{x_{j}}\left(T_{a_{j, k}}^{m} \partial_{x_{k}} v_{h}\right)+\Phi_{\gamma}^{\prime}(\gamma(T-t)) v_{h}\right\|_{L^{2}}^{2}\right. \\
\left.\quad+\left(\gamma \Phi_{\gamma}^{\prime \prime}(\gamma(T-t))-C\left(2^{4 h} \min \left\{2^{-2 h \alpha}, t^{\alpha-1} \mu\left(2^{-2 h}\right)\right\}+2^{2 h}\right)\right)\left\|v_{h}\right\|_{L^{2}}^{2}\right) d t \\
\geq \int_{0}^{\frac{T}{2}}\left(\left(\left\|\sum_{j, k=1}^{n} \partial_{x_{j}}\left(T_{a_{j, k}}^{m} \partial_{x_{k}} v_{h}\right)\right\|_{L^{2}}-\Phi_{\gamma}^{\prime}(\gamma(T-t))\left\|v_{h}\right\|_{L^{2}}\right)^{2}\right. \\
\left.\quad+\left(\gamma \Phi_{\gamma}^{\prime \prime}(\gamma(T-t))-C\left(2^{4 h} \min \left\{2^{-2 h \alpha}, t^{\alpha-1} \mu\left(2^{-2 h}\right)\right\}+2^{2 h}\right)\right)\left\|v_{h}\right\|_{L^{2}}^{2}\right) d t .
\end{aligned}
$$

From (27) it is possible to deduce that

$$
\left\|\sum_{j, k=1}^{n} \partial_{x_{j}}\left(T_{a_{j, k}}^{m} \partial_{x_{k}} v_{h}\right)\right\|_{L^{2}} \geq \frac{\lambda_{0}}{8} 2^{2 h}\left\|v_{h}\right\|_{L^{2}} .
$$

Suppose first that

$$
\Phi_{\gamma}^{\prime}(\gamma(T-t)) \leq \frac{\lambda_{0}}{16} 2^{2 h}
$$

From (27) we have

$$
\left\|\sum_{j, k=1}^{n} \partial_{x_{j}}\left(T_{a_{j, k}}^{m} \partial_{x_{k}} v_{h}\right)\right\|_{L^{2}}-\Phi_{\gamma}^{\prime}(\gamma(T-t))\left\|v_{h}\right\|_{L^{2}} \geq \frac{\lambda_{0}}{16} 2^{2 h}\left\|v_{h}\right\|_{L^{2}}
$$

and then, using also (16), we obtain 


$$
\begin{aligned}
& \int_{0}^{\frac{T}{2}}\left\|\partial_{t} v_{h}+\sum_{j, k=1}^{n} \partial_{x_{j}}\left(T_{a_{j, k}}^{m} \partial_{x_{k}} v_{h}\right)+\Phi_{\gamma}^{\prime}(\gamma(T-t)) v_{h}\right\|_{L^{2}}^{2} d t \\
& \geq \int_{0}^{\frac{T}{2}}\left(\left(\left\|\sum_{j, k=1}^{n} \partial_{x_{j}}\left(T_{a_{j, k}}^{m} \partial_{x_{k}} v_{h}\right)\right\|_{L^{2}}-\Phi_{\gamma}^{\prime}(\gamma(T-t))\left\|v_{h}\right\|_{L^{2}}\right)^{2}\right. \\
& \left.\quad+\left(\gamma \Phi_{\gamma}^{\prime \prime}(\gamma(T-t))-C\left(2^{4 h} \min \left\{2^{-2 h \alpha}, t^{\alpha-1} \mu\left(2^{-2 h}\right)\right\}+2^{2 h}\right)\right)\left\|v_{h}\right\|_{L^{2}}^{2}\right) d t \\
& \geq \int_{0}^{\frac{T}{2}}\left(\left(\frac{\lambda_{0}}{16} 2^{2 h}\right)^{2}+\gamma\left(\frac{T}{2}\right)^{\alpha-1}-C\left(2^{(4-2 \alpha) h}\right)\right)\left\|v_{h}\right\|_{L^{2}}^{2} d t .
\end{aligned}
$$

Then, there exist $\gamma_{0}>0$ and $C>0$ such that, for all $\gamma>\gamma_{0}$ and for all $h \geq 1$,

$$
\begin{aligned}
& \int_{0}^{\frac{T}{2}}\left\|\partial_{t} v_{h}+\sum_{j, k=1}^{n} \partial_{x_{j}}\left(T_{a_{j, k}}^{m} \partial_{x_{k}} v_{h}\right)+\Phi_{\gamma}^{\prime}(\gamma(T-t)) v_{h}\right\|_{L^{2}}^{2} d t \\
& \geq C \int_{0}^{\frac{T}{2}}\left(\gamma+\gamma^{\frac{1}{2}} 2^{2 h}\right)\left\|v_{h}\right\|_{L^{2}}^{2} d t
\end{aligned}
$$

Suppose finally that

$$
\Phi_{\gamma}^{\prime}(\gamma(T-t)) \geq \frac{\lambda_{0}}{16} 2^{2 h}
$$

From (15), the fact that $\lambda_{0} \leq 1$ and the properties of the modulus of continuity $\mu$ we obtain

$$
\begin{aligned}
\Phi^{\prime \prime}(\gamma(T-t)) & =t^{\alpha-1}\left(\Phi_{\gamma}^{\prime}(\gamma(T-t))\right)^{2} \mu\left(\frac{1}{\left.\Phi_{\gamma}^{\prime}(\gamma(T-t))\right)}\right) \\
& \geq t^{\alpha-1}\left(\frac{\lambda_{0}}{16}\right)^{2} 2^{4 h} \mu\left(\frac{16}{\lambda_{0}} 2^{-2 h}\right) \geq t^{\alpha-1}\left(\frac{\lambda_{0}}{16}\right)^{2} 2^{4 h} \mu\left(2^{-2 h}\right) .
\end{aligned}
$$

and

$$
\begin{aligned}
\Phi^{\prime \prime}(\gamma(T-t)) & =t^{\alpha-1}\left(\Phi_{\gamma}^{\prime}(\gamma(T-t))\right)^{2} \mu\left(\frac{1}{\left.\Phi_{\gamma}^{\prime}(\gamma(T-t))\right)}\right) \\
& =t^{\alpha-1} \Phi_{\gamma}^{\prime}(\gamma(T-t)) \frac{\mu\left(\frac{1}{\Phi_{\gamma}^{\prime}(\gamma(T-t))}\right)}{\frac{1}{\Phi_{\gamma}^{\prime}(\gamma(T-t))}} \geq\left(\frac{T}{2}\right)^{\alpha-1} .
\end{aligned}
$$

Consequently

$$
\begin{aligned}
& \int_{0}^{\frac{T}{2}}\left\|\partial_{t} v_{h}+\sum_{j, k=1}^{n} \partial_{x_{j}}\left(T_{a_{j, k}}^{m} \partial_{x_{k}} v_{h}\right)+\Phi_{\gamma}^{\prime}(\gamma(T-t)) v_{h}\right\|_{L^{2}}^{2} d t \\
& \geq \int_{0}^{\frac{T}{2}}\left(\gamma \Phi_{\gamma}^{\prime \prime}(\gamma(T-t))-C\left(2^{4 h} \min \left\{2^{-2 h \alpha}, t^{\alpha-1} \mu\left(2^{-2 h}\right)\right\}+2^{2 h}\right)\right)\left\|v_{h}\right\|_{L^{2}}^{2} d t \\
& \geq \int_{0}^{\frac{T}{2}}\left(\frac{\gamma}{2}\left(t^{\alpha-1}\left(\frac{\lambda_{0}}{16}\right)^{2} 2^{4 h} \mu\left(2^{-2 h}\right)+\left(\frac{T}{2}\right)^{\alpha-1}\right)-C\left(t^{\alpha-1} 2^{4 h} \mu\left(2^{-2 h}\right)+2^{2 h}\right)\right)\left\|v_{h}\right\|_{L^{2}}^{2} d t .
\end{aligned}
$$

Then, there exist $\gamma_{0}>0$ and $C>0$ such that, for all $\gamma>\gamma_{0}$ and for all $h \geq 1$, 


$$
\begin{gathered}
\int_{0}^{\frac{T}{2}}\left\|\partial_{t} v_{h}+\sum_{j, k=1}^{n} \partial_{x_{j}}\left(T_{a_{j, k}}^{m} \partial_{x_{k}} v_{h}\right)+\Phi_{\gamma}^{\prime}(\gamma(T-t)) v_{h}\right\|_{L^{2}}^{2} d t \\
\geq C \gamma \int_{0}^{\frac{T}{2}}\left(1+2^{2 h}\right)\left\|v_{h}\right\|_{L^{2}}^{2} d t
\end{gathered}
$$

As a conclusion, from (34), (36) and (37), there exist $\gamma_{0}>0$ and $C>0$ such that, for all $\gamma>\gamma_{0}$ and for all $h \in \mathbb{N}$,

$$
\begin{gathered}
\int_{0}^{\frac{T}{2}}\left\|\partial_{t} v_{h}+\sum_{j, k=1}^{n} \partial_{x_{j}}\left(T_{a_{j, k}}^{m} \partial_{x_{k}} v_{h}\right)+\Phi_{\gamma}^{\prime}(\gamma(T-t)) v_{h}\right\|_{L^{2}\left(\mathbb{R}^{n}\right)}^{2} d t \\
\geq C \int_{0}^{\frac{T}{2}}\left(\gamma+\gamma^{\frac{1}{2}} 2^{2 h}\right)\left\|v_{h}\right\|_{L^{2}}^{2} d t
\end{gathered}
$$

and (29) follows. The proof is complete.

\section{A counterexample}

Theorem 3 There exists

$$
l \in\left(\bigcap_{\alpha \in[0,1[} C^{0, \alpha}(\mathbb{R})\right) \cap C^{\infty}(\mathbb{R} \backslash\{0\})
$$

with

$$
\begin{gathered}
\frac{1}{2} \leq l(t) \leq \frac{3}{2}, \quad \text { for all } t \in \mathbb{R}, \\
\left|l^{\prime}(t)\right| \leq C_{\varepsilon}|t|^{-(1+\varepsilon)}, \quad \text { for all } \varepsilon>0 \text { and } t \in \mathbb{R} \backslash\{0\},
\end{gathered}
$$

and there exist $u, b_{1}, b_{2}, c \in C_{b}^{\infty}\left(\mathbb{R}_{t} \times \mathbb{R}_{x}^{2}\right)$, with

$$
\operatorname{Supp} u=\left\{(t, x) \in \mathbb{R}_{t} \times \mathbb{R}_{x}^{2} \mid t \geq 0\right\},
$$

such that

$$
\partial_{t} u+\partial_{x_{1}}^{2} u+l \partial_{x_{2}}^{2} u+b_{1} \partial_{x_{1}} u+b_{2} \partial_{x_{2}} u+c u=0 \quad \text { in } \mathbb{R}_{t} \times \mathbb{R}_{x}^{2} .
$$

Remark 5 Actually, the function $l$ will satisfy

$$
\sup _{t \neq 0}\left(\frac{|t|}{1+|\log | t||}\right)\left|l^{\prime}(t)\right|<+\infty .
$$

From (41) it is easy to obtain (40).

Proof We will follow the proof of Theorem 1 in [16] (see also Theorem 3 in [9]). Let $A, B, C, J$ be four $C^{\infty}$ functions, defined in $\mathbb{R}$, with 


$$
0 \leq A(s), B(s), C(s) \leq 1 \quad \text { and } \quad-2 \leq J(s) \leq 2, \quad \text { for all } s \in \mathbb{R}
$$

and

$$
\begin{array}{ll}
A(s)=1, \quad \text { for } s \leq \frac{1}{5}, & A(s)=0, \quad \text { for } s \geq \frac{1}{4}, \\
B(s)=0, \quad \text { for } s \leq 0 \text { or } s \geq 1, & B(s)=1, \text { for } \frac{1}{6} \leq s \leq \frac{1}{2}, \\
C(s)=0, \quad \text { for } s \leq \frac{1}{4}, & C(s)=1, \text { for } s \geq \frac{1}{3}, \\
J(s)=-2, \quad \text { for } s \leq \frac{1}{6} \text { or } s \geq \frac{1}{2}, & J(s)=2, \text { for } \frac{1}{5} \leq s \leq \frac{1}{3} .
\end{array}
$$

Let $\left(a_{n}\right)_{n},\left(z_{n}\right)_{n}$ be two real sequences such that

$$
\begin{aligned}
& -1<a_{n}<a_{n+1}, \quad \text { for all } n \geq 1, \quad \text { and } \quad \lim _{n} a_{n}=0, \\
& 1<z_{n}<z_{n+1}, \quad \text { for all } n \geq 1, \quad \text { and } \quad \lim _{n} z_{n}=+\infty .
\end{aligned}
$$

We define

$$
\begin{gathered}
r_{n}=a_{n+1}-a_{n}, \\
q_{1}=0 \text { and } \quad q_{n}=\sum_{k=2}^{n} z_{k} r_{k-1}, \quad \text { for } n \geq 2, \\
p_{n}=\left(z_{n+1}-z_{n}\right) r_{n} .
\end{gathered}
$$

We require

$$
p_{n}>1, \quad \text { for all } n \geq 1 \text {. }
$$

We set

$$
\begin{array}{ll}
A_{n}(t)=A\left(\frac{t-a_{n}}{r_{n}}\right), & B_{n}(t)=B\left(\frac{t-a_{n}}{r_{n}}\right), \\
C_{n}(t)=C\left(\frac{t-a_{n}}{r_{n}}\right), & J_{n}(t)=J\left(\frac{t-a_{n}}{r_{n}}\right) .
\end{array}
$$

We define

$$
\begin{gathered}
v_{n}\left(t, x_{1}\right)=\exp \left(-q_{n}-z_{n}\left(t-a_{n}\right)\right) \cos \sqrt{z_{n}} x_{1}, \\
w_{n}\left(t, x_{2}\right)=\exp \left(-q_{n}-z_{n}\left(t-a_{n}\right)+J_{n}(t) p_{n}\right) \cos \sqrt{z_{n}} x_{2}, \\
u\left(t, x_{1}, x_{2}\right) \\
= \begin{cases}v_{1}\left(t, x_{1}\right), & \text { for } t \leq a_{1}, \\
A_{n}(t) v_{n}\left(t, x_{1}\right)+B_{n}(t) w_{n}\left(t, x_{2}\right)+C_{n}(t) v_{n+1}\left(t, x_{1}\right), & \text { for } a_{n} \leq t \leq a_{n+1}, \\
0, & \text { for } t \geq 0 .\end{cases}
\end{gathered}
$$

The condition

$$
\lim _{n} \exp \left(-q_{n}+2 p_{n}\right) z_{n+1}^{\alpha} p_{n}^{\beta} r_{n}^{-\gamma}=0, \quad \text { for all } \alpha, \beta, \gamma>0,
$$

implies that $u \in C_{b}^{\infty}\left(\mathbb{R}_{t} \times \mathbb{R}_{x}^{2}\right)$.

We define 


$$
l(t)= \begin{cases}1, & \text { for } t \leq a_{1} \text { or } t \geq 0 \\ 1+J_{n}^{\prime}(t) p_{n} z_{n}^{-1}, & \text { for } a_{n} \leq t \leq a_{n+1}\end{cases}
$$

$l$ is a $C^{\infty}(\mathbb{R} \backslash\{0\})$ function. The condition

$$
\sup _{n}\left\{p_{n} r_{n}^{-1} z_{n}^{-1}\right\} \leq \frac{1}{2\left\|J^{\prime}\right\|_{L^{\infty}}}
$$

implies (39), i. e. the operator

$$
L=\partial_{t}-\partial_{x_{1}}^{2}-l(t) \partial_{x_{2}}^{2}
$$

is a parabolic operator. Moreover, $l$ is in $\bigcap_{\alpha \in[0,1[} C^{0, \alpha}(\mathbb{R})$ if

$$
\sup _{n}\left\{p_{n} r_{n}^{-1-\alpha} z_{n}^{-1}\right\}<+\infty, \quad \text { for all } \alpha \in[0,1[\text {. }
$$

Finally, we define

$$
\begin{aligned}
& b_{1}=-\frac{L u}{u^{2}+\left(\partial_{x_{1}} u\right)^{2}+\left(\partial_{x_{2}} u\right)^{2}} \partial_{x_{1}} u, \\
& b_{2}=-\frac{L u}{u^{2}+\left(\partial_{x_{1}} u\right)^{2}+\left(\partial_{x_{2}} u\right)^{2}} \partial_{x_{2}} u, \\
& c=-\frac{L u}{u^{2}+\left(\partial_{x_{1}} u\right)^{2}+\left(\partial_{x_{2}} u\right)^{2}} u .
\end{aligned}
$$

As in [16] and [9], the functions $b_{1}, b_{2}, c$ are in $C_{b}^{\infty}\left(\mathbb{R}_{t} \times \mathbb{R}_{x}^{2}\right)$ if

$$
\lim _{n} \exp \left(-p_{n}\right) z_{n+1}^{\alpha} p_{n}^{\beta} r_{n}^{-\gamma}=0, \quad \text { for all } \alpha, \beta, \gamma>0 .
$$

We choose, for $j_{0} \geq 2$,

$$
a_{n}=-e^{-\sqrt{\log \left(n+j_{0}\right)}}, \quad z_{n}=\left(n+j_{0}\right)^{3} .
$$

With this choice (42) and (43) are satisfied and we have

$$
r_{n} \sim e^{-\sqrt{\log \left(n+j_{0}\right)}} \frac{1}{\left(n+j_{0}\right) \sqrt{\log \left(n+j_{0}\right)}},
$$

where, for sequences $\left(f_{n}\right)_{n},\left(g_{n}\right)_{n}, f_{n} \sim g_{n}$ means $\lim _{n} \frac{f_{n}}{g_{n}}=\lambda$, for some $\lambda>0$. Similarly

$$
p_{n} \sim e^{-\sqrt{\log \left(n+j_{0}\right)}} \frac{n+j_{0}}{\sqrt{\log \left(n+j_{0}\right)}}
$$

and condition (44) is verified, for a suitable fixed $j_{0}$. Remarking that we have, for $j_{0}$ suitably large,

$$
q_{n}=\sum_{k=2}^{n} z_{k} r_{k-1} \geq z_{n} r_{n-1} \geq \lambda\left(n+j_{0}\right)^{\frac{7}{4}}
$$

and 


$$
p_{n} \leq \lambda\left(n+j_{0}\right)^{\frac{5}{4}}
$$

for some $\lambda>0$. Finally

$$
p_{n} r_{n}^{-1} z_{n}^{-1} \sim \frac{1}{n+j_{0}}
$$

As a consequence (45), (46), (47) and (48) are satisfied for a suitable fixed $j_{0}$. It remains to check (41). We have

$$
\left|l^{\prime}(t)\right| \leq\left\|J^{\prime \prime}\right\|_{L^{\infty}} p_{n} r_{n}^{-2} z_{n}^{-1}, \quad \text { for } a_{n} \leq t \leq a_{n+1}
$$

and consequently

$$
\begin{aligned}
\sup _{t \neq 0}\left(\frac{|t|}{1+|\log | t||}\right)\left|l^{\prime}(t)\right| & =\sup _{n} \sup _{t \in\left[a_{n}, a_{n+1}\right]}\left(\frac{|t|}{1+|\log | t||}\right)\left|l^{\prime}(t)\right| \\
& \leq \sup _{n}\left(\frac{a_{n}}{1-\log a_{n}}\right)\left\|J^{\prime \prime}\right\|_{L^{\infty}} p_{n} r_{n}^{-2} z_{n}^{-1} \\
& \leq C .
\end{aligned}
$$

The conclusion of the theorem is reached simply exchanging $t$ with $-t$.

Acknowledgements The first author is member of the "Gruppo Nazionale per l'Analisi Matematica, la Probabilità e le loro Applicazioni" (GNAMPA) of the "Istituto Nazionale di Alta Matematica" (INdAM). Both the authors thank the anonymous reviewer for her/his careful reading of the manuscript.

Funding Open access funding provided by Università degli Studi di Trieste within the CRUI-CARE Agreement.

Open Access This article is licensed under a Creative Commons Attribution 4.0 International License, which permits use, sharing, adaptation, distribution and reproduction in any medium or format, as long as you give appropriate credit to the original author(s) and the source, provide a link to the Creative Commons licence, and indicate if changes were made. The images or other third party material in this article are included in the article's Creative Commons licence, unless indicated otherwise in a credit line to the material. If material is not included in the article's Creative Commons licence and your intended use is not permitted by statutory regulation or exceeds the permitted use, you will need to obtain permission directly from the copyright holder. To view a copy of this licence, visit http://creativecommons.org/licenses/by/4.0/.

\section{References}

1. Bahouri, H., Chemin, J.Y., Danchin, R.: Fourier Analysis and Nonlinear Partial Differential Equations. Grundlehren der Mathematischen Wissenschaften, 343. Springer, Heidelberg (2011)

2. Bony, J.M.: Calcul Symbolique et propagation des singularités pour les équations aux dérivées partielles non linéaires. In: Annales Scientifiques de l'École Normale Supérieure, vol. 14, pp. 209-246 (1981)

3. Chemin, J.Y.: Fluides Parfaits Incompressibles. Astérisque, 230. Société Mathématique de France, Paris (1995)

4. Colombini, F., De Giorgi, E.; Spagnolo, S.: Sur les équations hyperboliques avec des coefficients qui ne déépendent que du temps. Ann. Scuola Norm. Sup. Pisa Cl. Sci. 6(3), 511-559 (1979) (French)

5. Colombini, F., Del Santo, D.; Kinoshita, T.: Well-posedness of the Cauchy problem for a hyperbolic equation with non-Lipschitz coefficients. Ann. Scula Norm. Super. Pisa Cl. Sci. 1(2), 327-358 (2002)

6. Colombini, F., Del Santo, D., Reissig, M.: On the optimal regularity of coefficients in hyperbolic Cauchy problems. Bull. Sci. Math. 127(4), 328-347 (2003) 
7. Colombini, F., Lerner, N.: Hyperbolic operators with non-Lipschitz coefficients. Duke Math. J. 77(3), 657-698 (1995)

8. Colombini, F., Métivier, G.: The Cauchy problem for wave equations with non Lipschitz coefficients; application to continuation of solutions of some nonlinear wave equations. Ann. Sci. Éc. Norm. Supér. 41(2), 177-220 (2008)

9. Del Santo, D., Prizzi, M.: Backward uniqueness for parabolic operators whose coefficients are nonLipschitz continuous in time. J. Math. Pures Appl. 84(4), 471-491 (2005)

10. Del Santo, D., Prizzi, M.: A new result on backward uniqueness for parabolic operators. Ann. Mat. Pura Appl. 194(2), 387-403 (2015)

11. Del Santo, D., Jäh, C.: Non-uniqueness and uniqueness in the Cauchy problem of elliptic and backward-parabolic equations. In: M. Ruzhansky, M. Reissig (eds.) Progress in Partial Differential Equations - Asymptotic Profiles, Regularity and Well-Posedness. Springer, Basel, pp. 27-52 (2013)

12. Gérard, P.: Rauch, J.: Propagation of the local regularity of solutions of nonlinear hyperbolic equations. Ann. Inst. Fourier (Grenoble) 37(3), 65-84 (1987)

13. Ghisi, M., Gobbino, M.: Finite vs infinite derivative loss for abstract wave equations with singular time-dependent propagation speed. Bull. Sci. Math. 166, 102918

14. Hörmander, L.: On the uniqueness of the Cauchy problem. II. Math. Scand. 7, 177-190 (1959)

15. Métivier, G.: Para-differential Calculus and Applications to the Cauchy Problem for Nonlinear Systems. Centro di Ricerca Matematica Ennio De Giorgi (CRM) Series, 5. Edizioni della Normale, Pisa (2008)

16. Pliś, A.: On non-uniqueness in Cauchy problem for an elliptic second order differential equation. Bull. Acad. Polon. Sci. Sèr. Sci. Math. Astronom. Phys. 11, 95-100 (1963)

17. Tarama, S.: Local uniqueness in the Cauchy problem for second order elliptic equations with nonLipschitzian coefficients. Publ. Res. Inst. Math. Sci. 33(1), 167-188 (1997)

18. Tarama, S.: Energy estimate for wave equations with coefficients in some Besov type class. Electron. J. Differ. Equ. 2007(85), 12 (2007)

Publisher's Note Springer Nature remains neutral with regard to jurisdictional claims in published maps and institutional affiliations. 